\title{
Identification of natural recombinants derived from PCV2a and PCV2b
}

\author{
J. Hu, S.L. Zhai, S.Y. Zeng, B.B. Sun, S.F. Deng, H.L. Chen, Y. Zheng, \\ H.X. Wang, X.P. Li, J.K. Liu, S. Cheng, X. Zhou, J.Q. Zhai and M.L. Luo \\ College of Veterinary Medicine, South China Agricultural University, \\ Guangzhou, Guangdong, China \\ Corresponding author: M.L. Luo \\ E-mail: luoml@scau.edu.cn
}

Genet. Mol. Res. 14 (4): 11780-11790 (2015)

Received December 8, 2014

Accepted May 18, 2015

Published October 2, 2015

DOI http://dx.doi.org/10.4238/2015.October.2.12

\begin{abstract}
Porcine circovirus type 2 (PCV2) is considered to be the main pathogen in $\mathrm{PC}$-associated diseases, and significantly affects the global pig-producing industry. PCV2 continuously evolves by point mutations and genome recombinations. In the present study, we aimed to further identify recombinant PCV2 strains. We used polymerase chain reaction to detect PCV2 in the carcasses of pigs with suspected infections from different regions of Guangdong Province in China. DNA was extracted from samples with confirmed infection and fullgenome amplification, sequencing, phylogenetic tree construction, gene recombination detection, and sequence alignment were performed in gene recombination analysis. Our results show that recombination occurred between the strains SHC (DQ104421) and ZhuJi2003 (AY579893). The recombination resulted in three recombinants: GD003 (KM503044), GD005 (KM487708), and GD008 (KM487709). Further analyses revealed that these novel recombinants appeared to result from recombination between the PCV2a and PCV2b strains, with crossover regions located in $O R F 2$. This study was a comprehensive analysis that used several different methods, which demonstrated that a cluster of PCV2 strains resulted from the same type of inter-genotypic
\end{abstract}


recombination pattern, with a breakpoint in the structural protein coding region. The results of our study provide both information on the recombination mechanism and disease pathogenesis and useful data for the prevention of PCV2 in the swine industry.

Key words: Swine industry; Genetic diversity; PCV2; Genome evolution; Genome recombination

\section{INTRODUCTION}

Porcine circovirus (PCV) belongs to the Circovirus genus of the Circoviridae family, and is approximately $17 \mathrm{~nm}$ in diameter very small-sized and single-stranded. PCV members include PCV1 and PCV2, which have circular genomes of 1759 and 1766 to 1768 nucleotides (nt), respectively (Shang et al., 2009). PCV1 is not pathogenic (Tischer et al., 1987), whereas PCV2 is closely associated with a multisystemic wasting syndrome, dyspnea, enteritis, breeding difficulty, porcine dermatitis, and nephropathy syndrome (Gillespie et al., 2009). As a result, PCV2 has attracted considerable attention in most swine-producing countries.

In general, the PCV2 genome size is 1767 or 1768 bp. However, in recent years, PCV2 with a 1766-bp genome has been reported (Guo et al., 2010). PCV1 and PCV2 share a similar genomic structure, with each containing three major open reading frames (ORF). ORF1, with $945 \mathrm{bp}$, is located in the coding region of the clockwise sense chain of the viral genome (nt 51 to 995) and encodes two replication proteins (Rep and Rep'). ORF2, with 702 or 705 bp, is located in the coding region of the counter-clockwise anti-sense chain of the viral genome (nt $1734 / 1735$ to 1030/1033/1034) and encodes the capsid protein (Cap). ORF3, with $315 \mathrm{bp}$ (nt 671 to 357), is embedded within ORF1 and encodes the ORF3 protein, which is not involved in viral replication but is associated with cell apoptosis (Cheung, 2003; Liu et al., 2007; Juhan et al., 2010). Studies of the PCV2 genome have revealed three PCV2 genotypes, namely PCV2a, PCV2b, and PCV2c; PCV2c has been reported only in Denmark (Cheung et al., 2007; Olvera et al., 2007). Genotyping of PCV2a and PCV2b is mainly based on the sequence of a specific fragment in ORF2. The specific motif in PCV2a is 1486 ACC/AAC/AAA/AT, and in PCV2b, it is $1486 \mathrm{TCA} / \mathrm{AAC} / \mathrm{CCC} / \mathrm{CGC}$ (Cheung et al., 2007). In addition, the full length of the PCV2a genome (1768 bp) differs from that of the PCV2b genome (1767 bp). According to phylogenetics, PCV2a is divided into five sub-groups, namely $2 \mathrm{~A}, 2 \mathrm{~B}, 2 \mathrm{C}, 2 \mathrm{D}$, and $2 \mathrm{E}$, and PCV2b is divided into the sub-groups 1A, 1B, and 1C (Grau-Roma et al., 2008; Segales et al., 2008).

Previous studies have revealed that PCV2 evolves through point mutation and genetic recombination, resulting in the diversity of PCV2 genes (Hesse et al., 2008; Wang et al., 2009). Analysis of the PCV2 genome demonstrates a high mutation rate in ORF2 (Olvera et al., 2007; Wang et al., 2009). The Cap protein contains different potential epitopes that may mutate under immune pressure, thereby increasing antigenicity. These findings support the significance of studying the genetics and evolution of ORF2. In addition, genetic recombination between strains of different subtypes, or within the same subtype, could generate new virus strains. Mixed infection provides the prerequisite for genetic recombination. Recent studies have reported a high frequency of mixed PCV2 infection with strains of different subtypes or the same subtype in swine (Hesse et al., 2008; Cai et al., 2011; Cadar et al., 2012). Previous studies have shown that genetic recombination occurred in ORF1 (Hesse et al., 2008; Kim et al., 2009), whereas subsequent studies discovered that ORF2 also contains sites of 
genetic recombination (Cai et al., 2012; Huang et al., 2013). Cadar et al. (2012) reported the occurrence of genetic recombination in ORF1 or ORF2 of PCV2a and PCV2b, discovered the recombinant virus between two strains of PCV2b, and that the recombination site is located in ORF1 and ORF2 (Cai et al., 2012). Huang et al. (2013) found 13 recombinant viral strains that were derived from PCV2a and PCV2b, with different patterns occurring in ORF2. Although diverse genetic recombination was detected, most cases occurred among strains of PCV2b; much less natural genetic recombination was detected between PCV2a and PCV2b. Because PCV2 evolves over time, much can be discovered regarding its recombination mechanism.

In the present study, molecular methods were used to analyze three suspected PCV2 recombinant virus strains isolated from swine samples. Our results identified the three strains as recombinant viruses. Chiasmata recombination was found between the ORF2 of PCV2a and PCV2b by the construction of a phylogenetic tree, recombinant detection, and sequence alignment, which resulted in the identification of three recombinant strains and enriched the PCV2 recombinant database. Our study provides data that may further extend our understanding of PCV2 recombination, epidemiological investigation, and vaccine development.

\section{MATERIAL AND METHODS}

\section{Sample collection}

Twenty-five samples of lymph nodes and spleens were collected from dead pigs from different commercial farms in Pingzhou, Yangjiang, Qingyuan, Jiangmen, and Zhaoqing in Guangdong Province, China, between 2013 and 2014. The pigs were possibly infected by PCV2 and had severe clinical lesions.

\section{PCV2 detection}

Total viral DNA was extracted from the collected samples in accordance with the manufacturer instructions (OMEGA, USA). PCV2 detection was performed using polymerase chain reaction (PCR) with 5'-AGAAGGGCTGGGTTATG-3' as the sense primer and 5'-TATCCAAGGAGGCGTTAC-3' as the reverse primer. The PCR conditions consisted of an initial step at $94^{\circ} \mathrm{C}$ for $3 \mathrm{~min} ; 35$ cycles at $94^{\circ} \mathrm{C}$ for $30 \mathrm{~s}, 55^{\circ} \mathrm{C}$ for $30 \mathrm{~s}$, and $72^{\circ} \mathrm{C}$ for $2 \mathrm{~min}$; and a final extension step at $72^{\circ} \mathrm{C}$ for $10 \mathrm{~min}$. The amplification products were separated by $1 \%$ agarose gel electrophoresis and photographed (GSG-2000 Nucleic acid/protein gel image analysis system; Hema Medical Instrument Co., Ltd, Zhuhai, China). The fragment size of a positive PCV2 sample was $460 \mathrm{bp}$.

\section{PCV2 amplification and sequence determination}

Eighteen samples from eight farms were determined to be positive for PCV2. These samples were used for genome amplification and sequencing using a method described in the literature (Huang et al., 2013). The complete sequence was amplified with 5'-TAGCCGCGGG CTGGCTGAACTTTTGA-3' (sense primer) and 5'-AGACCGCGGAAATTTCTGACAAAC G-3' (reverse primer). A reaction mixture of $25 \mu \mathrm{L}$ contained $2.5 \mu \mathrm{L} 10 \mathrm{X}$ PCR buffer, $2 \mu \mathrm{L}$ dNTP mix (2.5 mM each), $1 \mu \mathrm{L}$ of primers $(10 \mathrm{pmol} / \mu \mathrm{L}), 1.5 \mu \mathrm{L}$ DNA, $0.25 \mu \mathrm{L}$ TransTaq $^{\circledR}$ High Fidelity DNA Polymerase (TransGen, China), and double-distilled water. The PCR con- 
ditions were $94^{\circ} \mathrm{C}$ for $3 \mathrm{~min}$; followed by 30 cycles at $94^{\circ} \mathrm{C}$ for $30 \mathrm{~s}, 55^{\circ} \mathrm{C}$ for $30 \mathrm{~s}$, and $72^{\circ} \mathrm{C}$ for $2 \mathrm{~min}$; and a 10 -min extension at $72^{\circ} \mathrm{C}$. The PCR products obtained were separated by $1 \%$ agarose electrophoresis, extracted with an E.Z.N.A. ${ }^{\circledR}$ Gel Extraction Kit (OMEGA), and ligated with a PMD19-T vector system (Takara, Japan). The recombinant plasmids were transformed into Escherichia coli DH5a-competent cells and then sequenced in positive clones by Invitrogen (Guangzhou, China). A consensus sequence was obtained for each genomic DNA using a method described in the literature (Cai et al., 2012).

\section{Phylogenetic tree analysis}

Alignment of the complete genomic sequences was conducted with 26 different PCV2 genotype reference sequences from GenBank (Thompson et al., 1994; Cai et al., 2012). A neighbor-joining tree was then constructed according to a method described in the literature (Tamura et al., 2011). A PCV1 isolate was included as an outlier (KJ408799).

\section{Recombination analysis}

To analyze the recombination patterns of the PCV2 genome sequences obtained in our study, putative parents were sought using a previously described method (Lim et al., 2011; Cai et al., 2012). Nucleotide identity between the putative recombinant clusters and their putative parents was calculated using the recombination analysis software SimPlot (version 3.5.1) (Lole et al., 1999). To obtain additional confirmation of the occurrence of recombination and the breakpoint of the possible recombinants, a base-by-base comparison was performed using the BioEdit package (version 7.2.5), in accordance with a previously described method (Hesse et al., 2008). Phylogenetic trees were constructed based on the fragments of the cluster strains and the putative parents using MEGA 6.06.

\section{Virus isolation}

After the PK-15 cells had reached confluence, the culture media were removed and the cells were washed three times with phosphate-buffered saline, digested with $0.25 \%$ trypsin solution, and suspended in Dulbecco's modified Eagle's medium. Fluid from samples identified as positive by PCR was sterilized with a $0.22-\mu \mathrm{m}$ filter membrane, synchronously seeded at $10 \%$ into maintenance media (with $2 \%$ fetal bovine serum) with D-glucosamine (final concentration of $3 \mathrm{mM}$ ), and cultured at $37^{\circ} \mathrm{C}$ and $5 \% \mathrm{CO}_{2}$ for $72 \mathrm{~h}$. After three freeze-thaw cycles at $-20^{\circ} \mathrm{C}$ and centrifugation at $2000 \mathrm{rpm}$ for $10 \mathrm{~min}$, the supernatant was harvested and the DNA was extracted for PCR detection. The procedures used were the same as those described above for PCV2 detection. The harvested supernatant was used to inoculate PK-15 cells, and PCR was performed on each generation of cells.

\section{RESULTS}

\section{Sample screening}

Eighteen positive genomic DNAs were screened, and PCV2 was present in $72 \%$ of the collected samples. These findings suggest a high infection rate of PCV2 in the dead pigs. 


\section{Sequencing and phylogenetics}

PCV2-positive strains were subjected to three cycles of isolation, amplification, and sequencing. A phylogenetic tree was built to ascertain the genetic relationships between the 18 strains; three sequences were loosely clustered to PCV2b-1C. To better understand the evolutionary relationships between these three sequences, a phylogenetic approach was used to compare the three PCV2 strains with selected classical PCV strains and several recombinant strains (Table 1). The phylogenetic tree revealed that the three strains (GD003, GD005, and GD008) and the reference PCV2 sequences were classified into five distinct genetic groups, based on an analysis of the complete genome (Figure 1). Our results suggest that the three strains are more closely associated with KC800635 than with the PCV2b group, or with the recombinants HQ395028 and KC800635. These findings indicate that this novel cluster resulted from recombination between PCV2a and PCV2b.

Table 1. Sequencing results of PCV2 strains in the current study and as reported in the literature.

\begin{tabular}{llcc}
\hline GenBank accession No. & Identification & Year & Genomic size (nt) \\
\hline KM487708 & GD005 & 2013 & 1767 \\
KM487709 & GD008 & 2013 & 1767 \\
KM503044 & GD003 & 2014 & 1767 \\
AY579893 & ZhuJi2003 & 2004 & 1767 \\
DQ104421 & SHC & 2005 & 1768 \\
KC800644 & SXXAA-01 & 2012 & 1767 \\
KC800635 & SXYNA-01 & 2013 & 1768 \\
HQ395028 & 09GS & 2012 & 1767 \\
\hline
\end{tabular}

\section{Recombination analysis}

To further confirm whether GD003, GD005, and GD008 are recombinants of PCV2a and PCV2b, DQ104421 (PCV2a) and AY579893 (PCV2b-1A) were selected as putative parents for a similarity analysis using SimPlot 3.5.1. The results show that the potential breakpoint was possibly located at position $1040 \mathrm{nt}$ (Figure 2). The three recombinant strains were more similar to DQ104421 (nt sequence similarity ranging from 97.5 to $97.9 \%$ ) and AY579893 (nt similarity of 95.9 to $96.2 \%$ ) before and after the breakpoint, respectively. In contrast, the three strains exhibited 90.8 to $90.9 \%$ and 94.4 to $94.5 \%$ similarity to the putative strains. A base-by-base comparison revealed consistency between the three strains and DQ104421 at 21 substitution positions before the breakpoint, and AY579893 at 36 substitution positions after the breakpoint (Figure 3). Furthermore, amino acid sequences identified as the Rep and Cap proteins were also detected. The results showed that the Rep protein of the three strains exhibited 97.8 to $98.7 \%$ and 95.2 to $96.2 \%$ similarity with DQ104421 and AY579893, respectively; the Cap protein exhibited 89.7 to $90.1 \%$ and 93.6 to $94.0 \%$ similarity to DQ104421 and AY579893, respectively. Moreover, full-length sequence analysis showed that the three strains had 99.5 to $99.8 \%$ similarity to KC800644. In addition, we constructed inconsistent phylogenetic trees of the regions before and after the $1040 \mathrm{nt}$ of the cluster strains, and a discrepancy was observed (Figure 4). All of these findings indicate that the novel cluster of the three strains in this study is the result of natural recombination between DQ104421 and AY579893 within ORF2. 


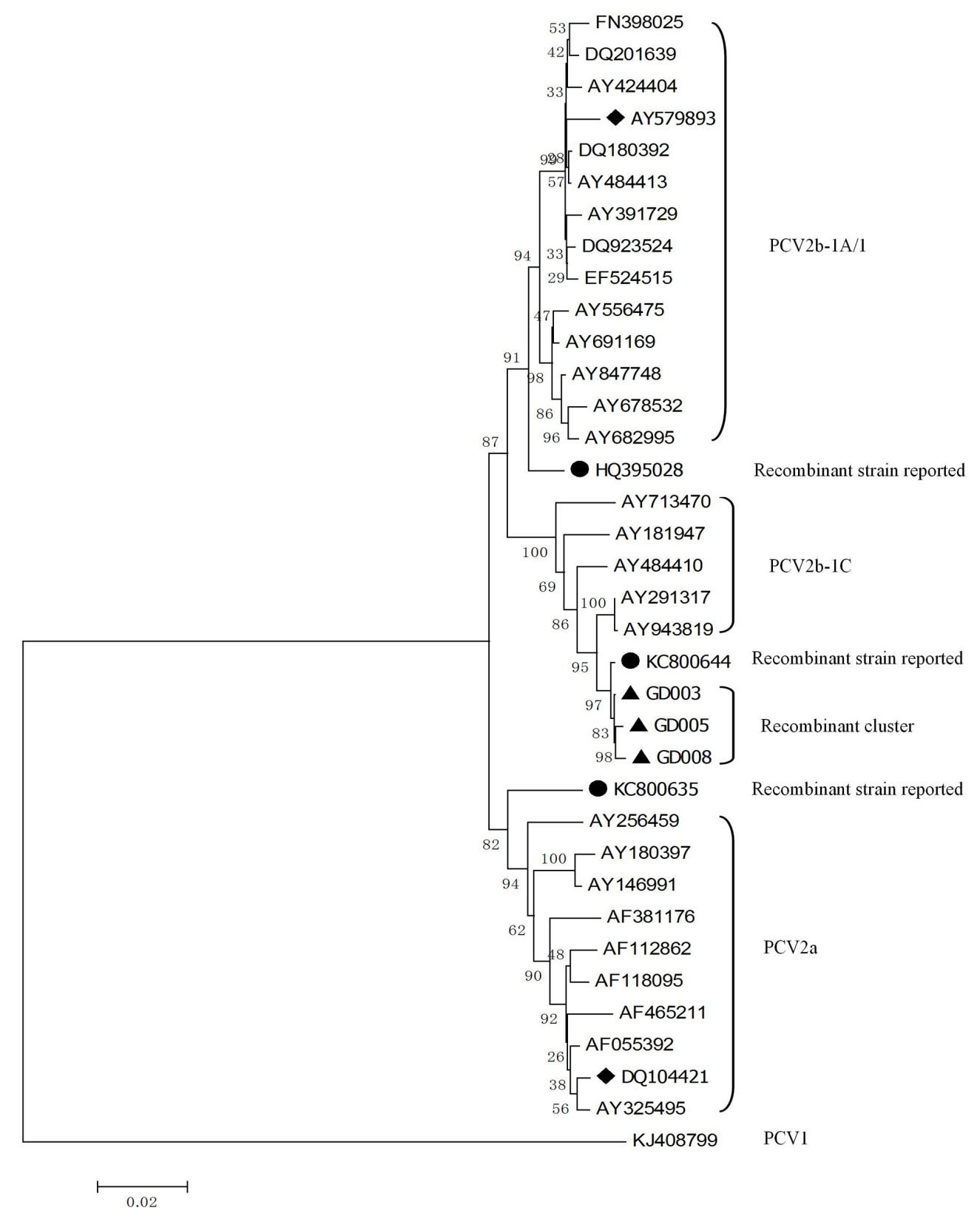

Figure 1. Phylogenetic analysis of the three novel strains and reference PCV2 sequences (GenBank), as analyzed by the full-genomic sequence. PCV1 isolate KJ408799 was included as an outgroup. The reliability of the tree was verified using a method described in the literature (1000 bootstrap replications; Huang et al., 2013). Black triangles indicate putative strains; black lozenges indicate putative parents DQ104421 (PCV2a) and AY579893 (PCV2b1A); black circles indicate recombinants. 


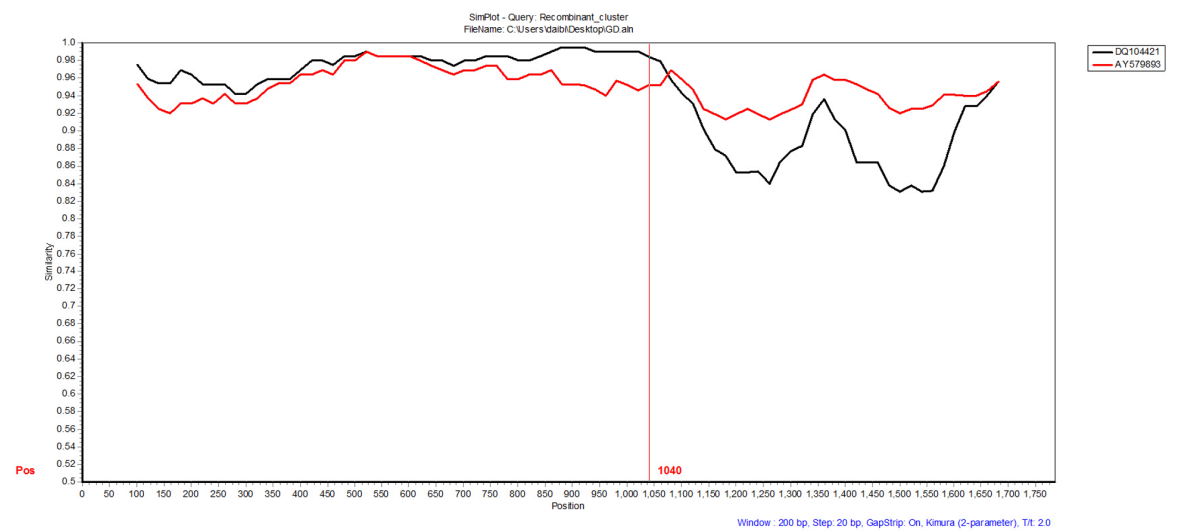

Figure 2. Recombination analysis of putative recombinant cluster viruses (GD003, GD005, and GD008) using the recombination analysis software SimPlot (version 3.5.1). The full-length PCV2 genome similarity between the cluster strains and the putative parents (DQ104421 and AY579893) were calculated using the Kimura twoparameter method with a transition-transversion ratio of 2 . The $y$-axis depicts the percentage of identity within a sliding window that is 200-bp wide and centered on the position plotted, with a step size between plots of $20 \mathrm{bp}$. The red vertical line indicates the potential breakpoint.

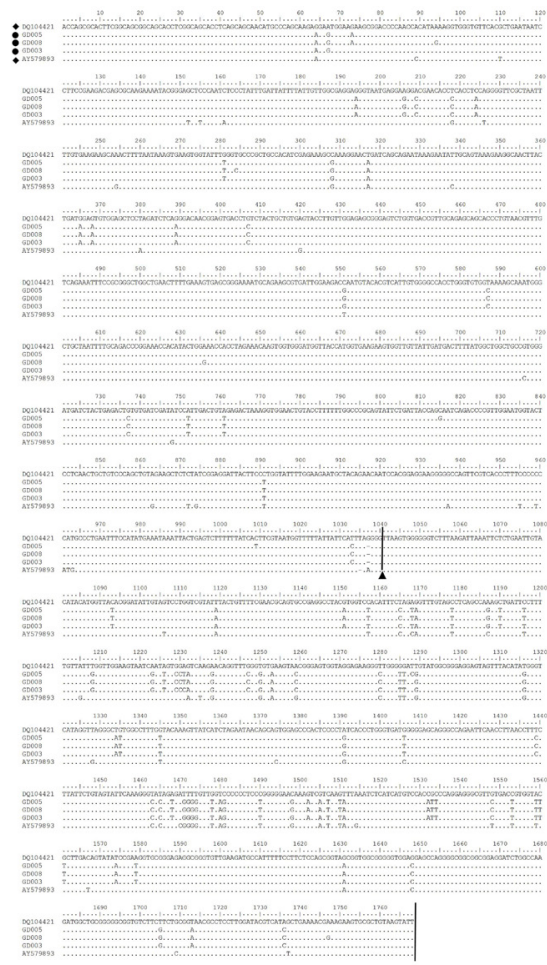

Figure 3. Complete genomic sequence comparison of GD003, GD005, GD008, and their putative parents. Black circles indicate recombinants; black lozenges indicate parental strains; black triangle indicates the possible breakpoint. Nucleotide sequences between the two black vertical lines show a higher similarity with AY579893, and nucleotide sequences outside the lines show a higher similarity with DQ104421 (PCV2a). 


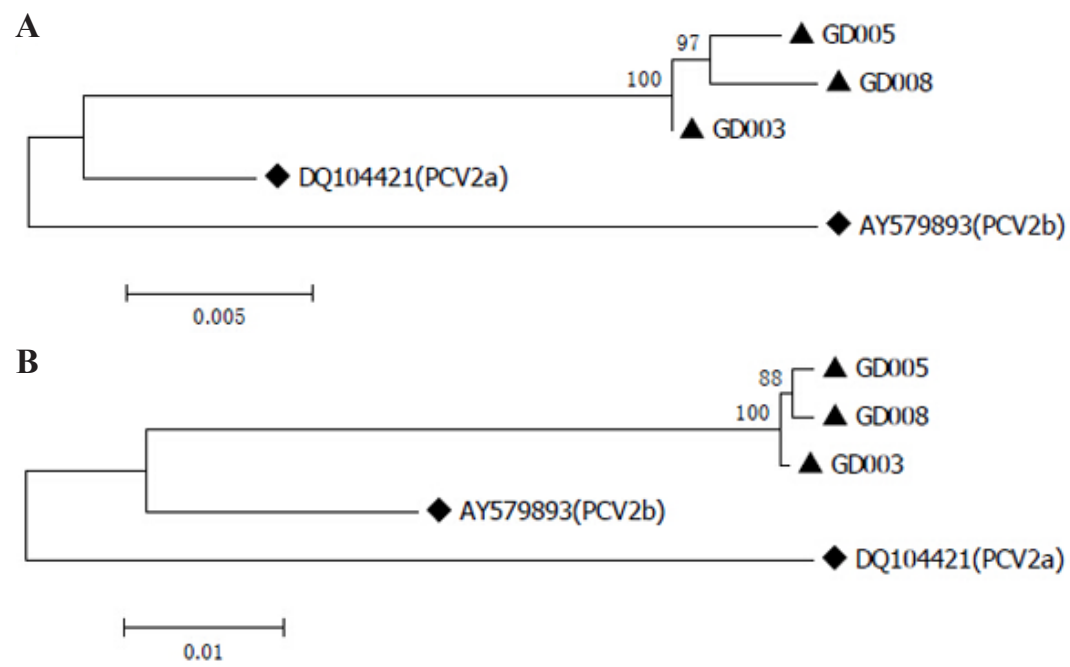

Figure 4. Phylogenetic analysis of the cluster strains GD003, GD005, GD008, and the putative parents DQ104421 and AY579893, according to genome segments (A) before and (B) after the breakpoint. Black triangles indicate recombinants; black lozenges indicate parents.

\section{Virus isolation}

The PCV2 strains were cultivated for six generations. Subsequently, PCR, sequence determination, and indirect immunofluorescence were performed to detect their proliferation. The outcomes were consistent with those of the first generation. All of our experimental findings indicate that the three recombinants can be stably cultivated in PK-15 cells.

\section{DISCUSSION}

PCV2a and PCV2b genotypes are widely distributed in the world (Allan et al., 1994), and the majority appear to convert from PCV2a to PCV2b (Wang et al., 2009). PCV2 genetically evolves by point mutation and genetic recombination. Since 2000, reports on PCV2 genetic variation have gradually increased. Point mutation is the primary method of full-genome evolution, because few studies have reported genomic recombination.

Since 2007, genetic recombination between strains of the same genotype or of different genotypes of PCV2 has been reported in several countries, including China (Hesse et al., 2008; Cheung, 2009; Cai et al., 2011; Huang et al., 2013). Analyses of genetic mutations in prevalent PCV2 strains have shown that mixed infections of strains of the same genotype or of different genotypes are present in swine (Zhai et al., 2011), which provides the prerequisite for natural recombination between different genotypes. In our study, tissue samples were collected from the carcasses of pigs with suspected infection from commercial hoggeries in Guangdong, and viral detection was then performed. Subsequently, gene amplification and full-genome sequencing were performed to analyze the genetic evolution of PCV2, in which three recombinant viral strains were discovered. The results of genetic recombinant analysis, based on phylogenetic tree construction, recombinant detection, and sequence alignment of the three suspected PCV2 strains, demonstrate that the genomes of these three strains are 
derived from chiasmata between PCV2a and PCV2b. Cai et al. (2012) found five recombinant viral strains in 40 samples that were collected from 40 commercial hoggeries from 2009 to 2010 in mainland China. These strains were derived from the same parent with the same recombination pattern. From 2012 to 2014, Huang et al. (2013) collected 141 samples from hoggeries in Shanxi Province and detected 47 samples with a mixed infection of PCV2a and PCV2b. Among those samples, 13 recombinant viruses were found, indicating that these recombinant viral strains were derived from the same parent with two recombination patterns. The results of our study are consistent with those of the previous studies, which confirm the incidence of novel viral strains through natural recombination between PCV2a and PCV2b.

The three recombinant viral strains (GD003, GDO005, and GD008) were classified as PCV2b, with an ORF2 size of $705 \mathrm{bp}$, which contained one additional lysine and differed from the ORF2 of the parent generation. The recombinant site was located at nt 1040. In Huang et al.'s (2013) study, three recombinants were classified as PCV2a and shared the same ORF2 size (702 bp) with the parent generation, in which the recombinant site was located at nt 1034 to 1391 . The other 10 recons were classified as PCV2b and shared the same ORF2 size (702 bp) with the parent generation, in which the recombinant site was located at nt 1034 . The five recons in Cai's study (2012) were identified to have a genotype resulting from recombination between PCV2a and PCV2b, with an ORF2 size of 705 bp, which was the same as that of one parent PCV2b (FJ948168) but differed from that of the other parent PCV2a (AY256459) (ORF2, 702 bp) by one additional lysine in the protein sequence. This recombinant site was located at nt 309 of ORF2 (nt 1338 of the full genome). The results of these studies indicate that ORF2 plays a significant role in the recombination of PCV2a and PCV2b, which generates new viral strains with different ORF2 sizes. ORF2 encodes proteins that are associated with viral structure and virulence, such as Cap (Horlen et al., 2008). The Cap protein is considered one of the major antigenic determinants, and functions efficiently in viral attachment to host cell surface receptors (Misinzo et al., 2006; Finsterbusch and Mankertz, 2009). Guo et al. (2011) reported that recombinant viruses obtained higher replication with antigenic drift. Another study showed that PCV2 DNA is present in human feces, human vaccines, beef, rodents, and even commercial pig vaccines (Zhai et al., 2014). An increasing number of studies report PCV2 mixed infections, with a high mutation rate in ORF2 and multiple potential epitopes in the Cap protein. With the selective pressure of vaccines, the virus could gradually evolve to generate several new viral strains. Therefore, the replication capacity, pathogenicity, and host range of PCV2 require further in-depth investigation.

The recombinants in our study were identified from samples collected only in Guangdong Province, whereas studies in other countries and regions have discovered other recombinants. These other studies indicate that recombination between PCV2a and PCV2b, or within PCV2b, are via chiasmata located within ORF1 or ORF2. In addition, recombination between PCV1 and PCV2a is based on sequence exchange between ORF1 and ORF2. Does recombination occur within PCV2a, or between PCV1 and PCV2b? What factors are associated with PCV2 evolution and recombination? Further investigations should include larger sample sizes.

The three recombinant viral strains in this study, GD003, GD005, and GD008, were derived from ORF2 chiasmata between PCV2a and PCV2b at nt 1040. The results of this study add to our knowledge of PCV recombination between strains of different genotypes, which may further extend our understanding of viral recombinant patterns, pathogenesis, and vaccine development. 


\section{Conflicts of interest}

The authors declare no conflict of interest.

\section{ACKNOWLEDGMENTS}

Research supported by grants from the University-Industry Cooperation Projects of the National Ministry of Education and Guangdong Province, China (\#2011A090200117).

\section{REFERENCES}

Allan GM, Phenix KV, Todd D and McNulty MS (1994). Some biological and physico-chemical properties of porcine circovirus. Zentralbl. Veterinarmed. B. 41: 17-26.

Cadar D, Csagola A, Lorincz M, Tombacz K, et al. (2012). Detection of natural inter- and intra-genotype recombination events revealed by cap gene analysis and decreasing prevalence of PCV2 in wild boars. Infect. Genet. Evol. 12: 420-427.

Cai L, Han X, Ni J, Yu X, et al. (2011). Natural recombinants derived from different patterns of recombination between two PCV2b parental strains. Virus Res. 158: 281-288.

Cai L, Ni J, Xia Y, Zi Z, et al. (2012). Identification of an emerging recombinant cluster in porcine circovirus type 2. Virus Res. 165: 95-102.

Cheung AK (2003). Transcriptional analysis of porcine circovirus type 2. Virology 305: 168-180.

Cheung AK (2009). Homologous recombination within the capsid gene of porcine circovirus type 2 subgroup viruses via natural co-infection. Arch. Virol. 154: 531-534.

Cheung AK, Lager KM, Kohutyuk OI, Vincent AL, et al. (2007). Detection of two porcine circovirus type 2 genotypic groups in United States swine herds. Arch. Virol. 152: 1035-1044.

Finsterbusch T and Mankertz A (2009). Porcine circoviruses - small but powerful. Virus Res. 143: 177-183.

Gillespie J, Opriessnig T, Meng XJ, Pelzer K, et al. (2009). Porcine circovirus type 2 and porcine circovirus associated disease. J. Vet. Intern. Med. 23: 1151-1163.

Grau-Roma L, Crisci E, Sibila M, Lopez-Soria S, et al. (2008). A proposal on porcine circovirus type 2 (PCV2) genotype definition and their relation with postweaning multisystemic wasting syndrome (PMWS) occurrence. Vet. Microbiol. 128: 23-35.

Guo LJ, Lu YH, Wei YW, Huang LP, et al. (2010). Porcine circovirus type 2 (PCV2): genetic variation and newly emerging genotypes in China. Virol. J. 7: 273.

Guo L, Lu Y, Wei Y, Huang L, et al. (2011). Porcine circovirus genotype 2a (PCV2a) and genotype 2b (PCV2b) recombinant mutants showed significantly enhanced viral replication and altered antigenicity in vitro. Virology 419: 57-63.

Hesse R, Kerrigan M and Rowland RR (2008). Evidence for recombination between PCV2a and PCV2b in the field. Virus Res. 132: 201-207.

Horlen KP, Dritz SS, Nietfeld JC, Henry SC, et al. (2008). A field evaluation of mortality rate and growth performance in pigs vaccinated against porcine circovirus type 2. J. Am. Vet. Med. Assoc. 232: 906-912.

Huang Y, Shao M, Xu X, Zhang X, et al. (2013). Evidence for different patterns of natural inter-genotype recombination between two PCV2 parental strains in the field. Virus Res. 175: 78-86.

Juhan NM, LeRoith T, Opriessnig T and Meng XJ (2010). The open reading frame 3 (ORF3) of porcine circovirus type 2 (PCV2) is dispensable for virus infection but evidence of reduced pathogenicity is limited in pigs infected by an ORF3-null PCV2 mutant. Virus Res. 147: 60-66.

Kim HK, Luo Y, Moon HJ, Park SJ, et al. (2009). Phylogenetic and recombination analysis of genomic sequences of PCV2 isolated in Korea. Virus Genes 39: 352-358.

Lim TH, Lee HJ, Lee DH, Lee YN, et al. (2011). An emerging recombinant cluster of nephropathogenic strains of avian infectious bronchitis virus in Korea. Infect. Genet. Evol. 11: 678-685.

Liu J, Zhu Y, Chen I, Lau J, et al. (2007). The ORF3 protein of porcine circovirus type 2 interacts with porcine ubiquitin E3 ligase Pirh2 and facilitates p53 expression in viral infection. J. Virol. 81: 9560-9567.

Lole KS, Bollinger RC, Paranjape RS, Gadkari D, et al. (1999). Full-length human immunodeficiency virus type 1 genomes from subtype C-infected seroconverters in India, with evidence of intersubtype recombination. J. Virol. 73: 152-160.

Misinzo G, Delputte PL, Meerts P, Lefebvre DJ, et al. (2006). Porcine circovirus 2 uses heparan sulfate and chondroitin sulfate B glycosaminoglycans as receptors for its attachment to host cells. J. Virol. 80: 3487-3494. 
Olvera A, Cortey M and Segales J (2007). Molecular evolution of porcine circovirus type 2 genomes: phylogeny and clonality. Virology 357: 175-185.

Segales J, Olvera A, Grau-Roma L, Charreyre C, et al. (2008). PCV-2 genotype definition and nomenclature. Vet. Rec. 162: 867-868.

Shang SB, Jin YL, Jiang XT, Zhou JY, et al. (2009). Fine mapping of antigenic epitopes on capsid proteins of porcine circovirus, and antigenic phenotype of porcine circovirus type 2. Mol. Immunol. 46: 327-334.

Tamura K, Peterson D, Peterson N, Stecher G, et al. (2011). MEGA5: molecular evolutionary genetics analysis using maximum likelihood, evolutionary distance, and maximum parsimony methods. Mol. Biol. Evol. 28: 2731-2739.

Thompson JD, Higgins DG and Gibson TJ (1994). CLUSTAL W: improving the sensitivity of progressive multiple sequence alignment through sequence weighting, position-specific gap penalties and weight matrix choice. Nucleic Acids Res. 22: 4673-4680.

Tischer I, Peters D, Rasch R and Pociuli S (1987). Replication of porcine circovirus: induction by glucosamine and cell cycle dependence. Arch. Virol. 96: 39-57.

Wang F, Guo X, Ge X, Wang Z, et al. (2009). Genetic variation analysis of Chinese strains of porcine circovirus type 2. Virus Res. 145: 151-156.

Zhai SL, Chen SN, Wei ZZ, Zhang JW, et al. (2011). Co-existence of multiple strains of porcine circovirus type 2 in the same pig from China. Virol. J. 8: 517.

Zhai SL, Chen SN, Xu ZH, Tang MH, et al. (2014). Porcine circovirus type 2 in China: an update on and insights to its prevalence and control. Virol. J. 11: 88. 